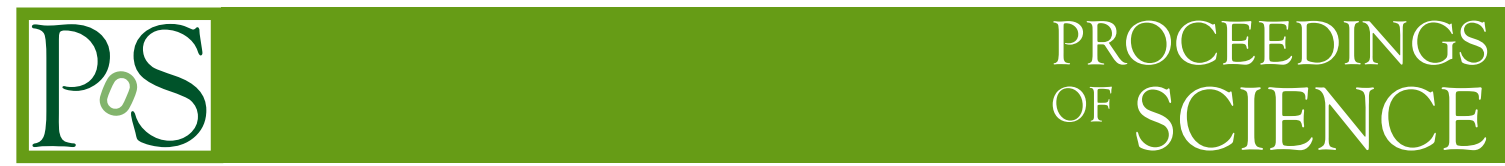

\title{
Kaon Physics from 30,000 Feet and Other Perspectives
}

\author{
Laurence Littenberg ${ }^{* \dagger}$ \\ Brookhaven National Laboratory \\ E-mail: littenbe@bnl.gov
}

Some things are seen better from a distance

2013 Kaon Physics International Conference

Apr 29-30 and May 1st

University of Michigan, Ann Arbor, Michigan - USA

\footnotetext{
${ }^{*}$ Speaker.

${ }^{\dagger}$ Communications with A. Buras, A. Ceccucci, S. Kettell, and T. Komatsubara are gratefully acknowledged.
} 

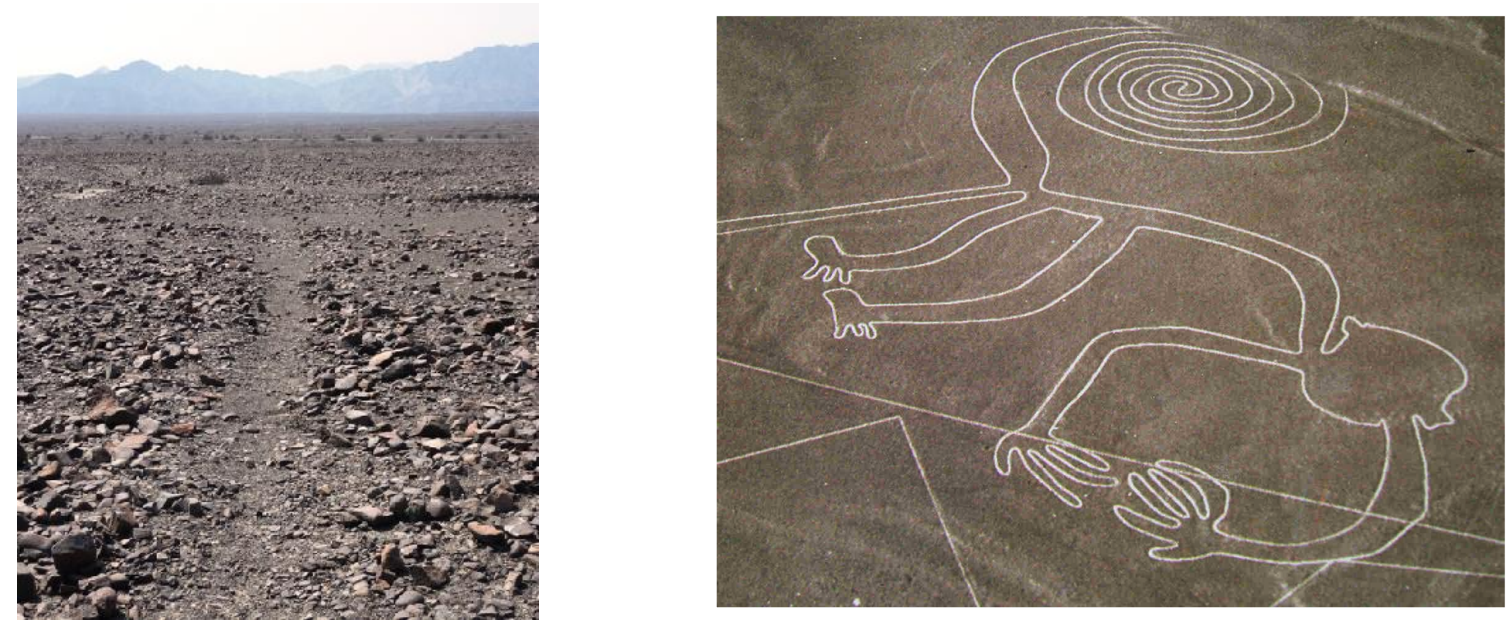

Figure 1: Nazca lines, left: from the ground, right: from the air.

Some things don't look like much from the ground but seen from high above, interesting patterns emerge. This can be seen very well in the case in the case of the Nazca lines in the high Peruvian desert (see Fig. 1). I want to convince you that this is true of rare kaon physics. The Nazca lines included figures such as triangles, although they never quite reached the triangle in Fig. 2. This is quite an old figure, although newer than the Nazca ones and it dates from a time when it seemed competitive to determine the CKM parameters from rare kaon decays. But the required experiments were very demanding, and people preferred to invest in B-factories. These were much more expensive but they held the promise of larger effects. The rare kaon experiments then became seen mainly as possible venues for discovering new physics, and indeed they are generically sensitive to extremely high mass scales.

\section{Discoveries}

But what does it take to make such a discovery? Over the past few years, the standard of reliability in such cases has evolved from perhaps $3 \sigma$ to $5 \sigma$ - that is, from a one in 770 chance of being a fluctuation to a one in 3.5 million chance. To put this in perspective, according to the National Highway Traffic Safety Administration, in 2011 there were 1.1 deaths per 100 million miles on US highways [1]. Thus if one drives 10,000 miles per year, one has about a 1 in 9000 chance of being killed. But most of us drive this far, so that we are willing to bet our lives on $3.7 \sigma$, whereas we demand $5 \sigma$ of our results. That's dedication!

It's instructive to ask how many events are required to establish a $5 \sigma$ discrepancy with the SM (Standard Model) expectation. To enforce uniformity in answering this question, I introduce the concept of "equivalent" events. This means the number of events that would establish a given uncertainty in a perfect experiment in which there was no background and no systematic errors. Thus in such an experiment 100 events would lead to a $\pm 10 \%$ relative error. However when the question of how probable the SM result is, it's the number of expected SM events that is the relevant one. Thus if for example one expects $100 \mathrm{SM}$ events and one sees 150 , one has a $5 \sigma$ discrepancy. Fig. 3 from a paper by Straub [2] shows how this works in practice. The plot shows the branching 


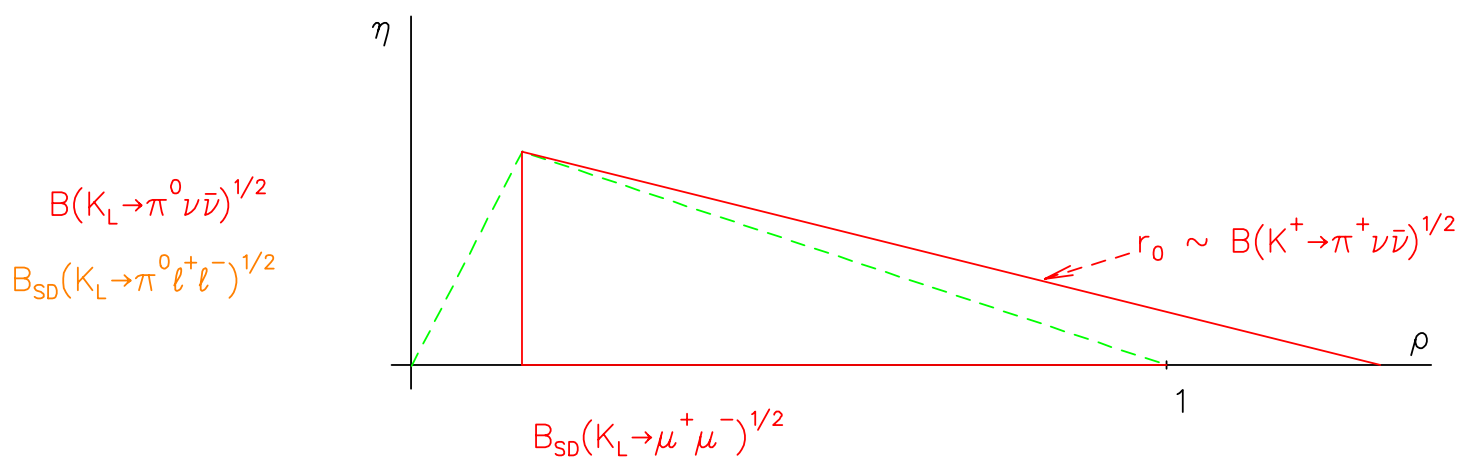

Figure 2: Two versions of the CKM triangle. The dashed green line shows the conventional triangle, while the solid red line shows a version obtained from rare kaon decays alone.

ratio of the neutral versus the charged $K \rightarrow \pi v \bar{v}$ in various models. The yellow star indicates the SM. Four BSM model predictions are shown in red, green, blue and brown (see Ref [2] for details). The black rectangles at the lower left indicate the $5 \sigma$ discovery power of experiments of successively greater sensitivity, corresponding to 10,100 , and 1000 effective events. It's apparent that even a 10 event experiment could probe a huge unexplored area on this plane at the $5 \sigma$ level. But the most interesting territory is that closest to the SM point because the phenomenological constraints for the various models are most easily satisfied there.

\section{A comment on the experimental challenge of $K \rightarrow \pi v \bar{v}$}

From the point of view of experiment, both the charged and neutral $K \rightarrow \pi v \bar{v}$ suffer from very low rates and very poor signatures. In the SM they are each have branching ratios of few times $10^{-11}$, and only one of the three decay products is detectable. In fact, the only reason for hope is that although their signatures are poor, the signatures of the primary backgrounds to them are rather good. So one can hope to recognize and reject background events. But to exploit this advantage very good veto rejection is required, typically $10^{4} /$ particle. 


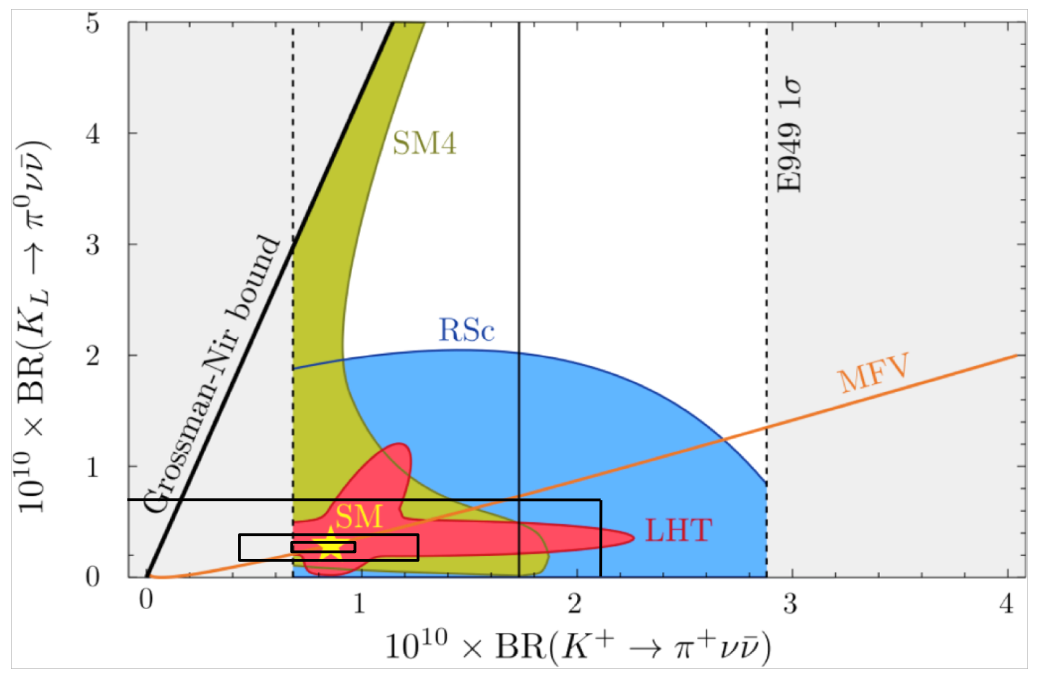

Figure 3: Predictions for $B\left(K_{L} \rightarrow \pi^{0} v \bar{v}\right)$ vs $B\left(K^{+} \rightarrow \pi^{+} v \bar{v}\right)$ in the SM and various BSM models (from Ref. [2]). Dashed vertical lines are the 1- $\sigma$ limits from E787/949. Rectangles at lower left indicate the limits of possible $5 \sigma$ discovery from successively 10, 100, and 1000 equivalent event experiments of both modes.

\section{A Whirlwind tour of the $K \rightarrow \pi v \bar{v}$ experiments}

There's a certain symmetry in searches for $K \rightarrow \pi v \bar{v}$. There's one each of charged and neutral searches in the last generation, BNL E787/949 [3] and KEK E391a [4], one each underway at the moment, CERN NA62 [5] and J-PARC's KOTO [6] and one each contemplated for the future, ORKA [7] at Fermilab and a yet-to-be-named KOPIO-like experiment at Project-X [8]. Each mode features two experimental techniques that have been used or proposed. A series of stopped$\mathrm{K}$ beam $K^{+} \rightarrow \pi^{+} v \bar{v}$ experiments culminating in the E787/E949 series resulted in the discovery of this mode. Seven events were observed but because of the background this corresponded to only two equivalent events. E949 was initially designed as a 10 equivalent event experiment but had very little running due to funding problems in HEP nationally. The proposed 1000-event ORKA experiment proposed for Fermilab is modeled closely on E787/949. It would improve on the former experiment by increasing the exposure and the acceptance each by a factor of 10 . By contrast the experiment presently underway at CERN, NA62, for the first time uses a highenergy in-flight technique. This experiment is designed to see 100 events in two years of running, now scheduled to begin in 2014. It uses an unseparated beam similar in instantaneous intensity 
and kaon fraction to that used almost 20 years ago by BNL E865 [9] to search for BSM decays such as $K^{+} \rightarrow \pi^{+} \mu^{+} e^{-}$at the $10^{-11}$ level. $K^{+} \rightarrow \pi^{+} v \bar{v}$ has a much poorer signature than the targets of E865, and the sensitivity goal is about an order of magnitude more ambitious so that many of the technical advances of subsequent years need to be deployed in the new experiment. By contrast the ORKA proposal reverts back to the stopped-K technique, exploiting the expertise gained in E787/949 and the more intense beam available at Fermilab to envision a 1000-event class experiment.

In the case of the neutral mode, the two techniques can be categorized as low-energy and highenergy. At low energy, it is possible to time the kaons and directionalize the gammas from the $\pi^{0}$ so that one can determine the kaon momentum and decay vertex, and thereby access the center of mass of the decay. At high energy one loses these abilities, but gains in vetoing power against the extra particles that characterize the background processes, and generally gains in gamma energy resolution. So far only the high energy technique has been used in actual experiments, but in fact the beam energy has been rather low. The one experiment to report results so far was E391a at the KEK PS [4] which established the current branching ratio upper limit of $2.6 \times 10^{-8}$ at $90 \% \mathrm{CL}$, had a $K_{L}$ momentum spectrum that peaks at around $1.7 \mathrm{GeV} / \mathrm{c}$. Much was learned from E391a and the apparatus was taken to J-PARC where far more intense beams are available, and very significantly upgraded in capability. That experiment, now christened KOTO, is currently taking physics data. Unfortunately, due to budgetary and space constraints, the neutral beam is taken off at $16^{\circ}$ instead of $4^{\circ}$, so that in spite of the higher primary proton energy, the kaon spectrum is even softer than at KEK. The plan is to eventually go to a smaller production angle so that the experiment can go from its present goal of discovering $K_{L} \rightarrow \pi^{0} v \bar{v}$ to studying it with a 100-event sample. The low energy technique, pioneered by the proponents of KOPIO [10] has never been tried in an actual experiment. The planned kaon beam at Project-X is a very promising venue for a KOPIO-like experiment. KOPIO at the BNL AGS was forced to extract a neutral beam at a very large angle in order to make it soft enough for timing to work. This in turn led to the need for a very large beam aperture with many attendant problems. This would not be necessary at Project-X. Moreover before its demise, many of the features of the KOPIO experimental technique were successfully tested at BNL. Primary beam bunching at the 200ps level was demonstrated [11], as well as the energy [12] and angular resolution necessary for photon detection [13]. Once in the $K_{L}$ center of mass, many handles for background rejection become available. KOPIO was designed as a 100 equivalent event exporiment. It appears quite possible to conceive of a 1000-event experiment of this type at Project-X.

\section{A few comments on other rare decays}

\section{1 $K_{L} \rightarrow \pi^{0} e^{+} e^{-}$and $K_{L} \rightarrow \pi^{0} \mu^{+} \mu^{-}$}

The decays $K_{L} \rightarrow \pi^{0} e^{+} e^{-}$and $K_{L} \rightarrow \pi^{0} \mu^{+} \mu^{-}$are very closely related to $K_{L} \rightarrow \pi^{0} v \bar{v}$ in the Standard Model but are sensitive to additional operators once one goes beyond it [14]. They are more tractable experimentally, but are also subject to certain difficult intrinsic backgrounds and suffer from long-distance contributions. There's a CP-conserving two-photon long-distance contribution which must be calculated, although insight into it can be gained from measurements 


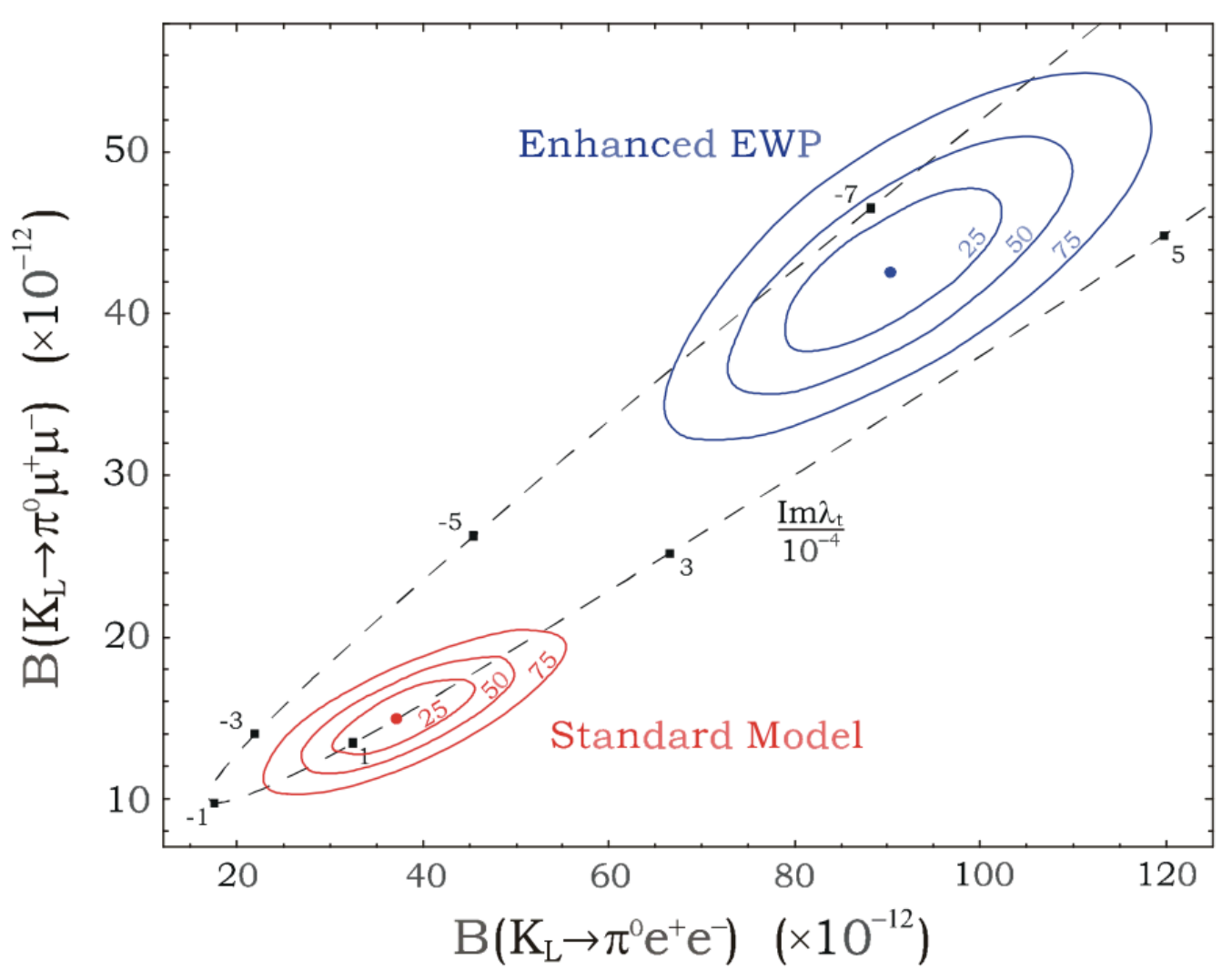

Figure 4: From Ref. [17], 25\%, 50\% and 75\% confidence-level regions for the Standard Model (assuming positive interference) and the enhanced-electroweak-penguin model of Ref. [12], taking into account all the present uncertainties..

of $K_{L} \rightarrow \pi^{0} \gamma \gamma$ and an order $\varepsilon$ CP-violating piece that can be determined from measurements of $K_{S} \rightarrow \pi^{0} e^{+} e^{-}$[15] and $K_{S} \rightarrow \pi^{0} \mu^{+} \mu^{-}$[16], modulo a sign. The sign is important since this contribution can interfere with the short-distance direct CP-violating one. These complications make it difficult to extract SM parameters from these decays, but BSM contributions can be large enough to be unambiguous in these decays [17]. See Fig. 4 for an example.

Ironically, the detection of a signal of the size of the enhanced EWP shown in Fig. 4 is not beyond the present state of the art. A decade ago $\mathrm{KTeV}$ achieved single event sensitivities of $1.04 \times 10^{-10}$ and $7.5 \times 10^{-11}$ for $K_{L} \rightarrow \pi^{0} e^{+} e^{-}$[18] and $K_{L} \rightarrow \pi^{0} \mu^{+} \mu^{-}$[19] respectively. These are respectively only factors 2.5 and 5 higher than the SM estimates shown in Fig. 4. The estimated residual background in the two modes was 0.99 and 0.37 so that the SM signal to background would have been $1: 2.5$ and $1: 1.9$, figures that are not very frightening in the context of modern collider physics analyses. KaMI [20], the proposed (but unfortunately not approved) successor to KTeV could have reached a s.e.s of $4 \times 10^{-13}$ in three years of running and could have seen $\sim 100$ S.M. $K_{L} \rightarrow \pi^{0} e^{+} e^{-}$events and $\sim 38 \mathrm{SM} K_{L} \rightarrow \pi^{0} \mu^{+} \mu^{-}$events. A signal as large as that the enhanced EWP of Fig. 4 would have been established as BSM by $5 \sigma$. 


\section{$4.2 K \rightarrow \mu^{+} \mu^{-}$}

$K_{L} \rightarrow \mu^{+} \mu^{-}$is unique among the ultra-rare kaon decays in that there is copious experimental data, with the most recent experiment having collected over 6000 events [21]. However the process is entirely dominated by a long distance contribution of the form $K_{L} \rightarrow \gamma \gamma \rightarrow \mu^{+} \mu^{-}$. The theoretical situation has been well-studied, most recently by Isidori and Unterdorfer [22], and I described the experimental issues involved in extracting the short distance component several years ago [23]. Using slightly updated input, one can extract the dispersive contribution, which includes the shortdistance piece as $(3.2 \pm 1.2) \times 10^{-10}$. Thus what started off as a 6200 -event result devolves into a mere 7-effective-event one. Moreover, one must calculate the long-distance part of the dispersive component, including the sign, in order to extract that short-distance piece. Most theorists agree it is not presently possible to make a precise calculation. Perhaps in a few years a lattice calculation could meet this challenge. This could motivate a more precise experiment.

There is a recent result from $\mathrm{LHCb}$ on $K_{S} \rightarrow \mu^{+} \mu^{-}$. This process is more tractable theoretically than the $K_{L}$ analogue, and its short-distance part is CP-violating in the $\mathrm{SM}$, as opposed to the case of $K_{L}$ in which it is CP-conserving. But the long-distance contributions are still about 25 times larger than the SM short-distance prediction. Furthermore, $K_{L} \rightarrow \mu^{+} \mu^{-}$gives an essentially flat (in time) background of about $3 \times 10^{-11}$ that would have to be measured and subtracted in a $K_{S}$ experiment. The current measurement of $K^{+} \rightarrow \pi^{+} \nu \bar{v}$ limits $K_{S} \rightarrow \mu^{+} \mu^{-}$less than about $10^{-11}$ in the SM and most BSM models. Thus any sensitivity exceeding this is interesting. The new $90 \%$ CL upper limit, $9 \times 10^{-9}$ [24], although a big improvement on previous work, is three orders of magnitude short of this.

\section{Final Thoughts}

There's a very poignant irony in ultra-rare kaon physics. Twenty-five years ago people considered whether the SM parameters could be extracted from these decays, particularly $K^{+} \rightarrow \pi^{+} v \bar{v}$ and $K_{L} \rightarrow \pi^{0} v \bar{v}$. But the focus stayed on the $B$ sector and B-factories were built and yielded fairly consistent results for these parameters. Meanwhile the rare $\mathrm{K}$ decays became seen as clean venues to search for significant BSM effects. However more recently as results have become more plentiful and more precise, problems have begun to show up. Although the details of the disagreements have changed over the last couple of years, the trend continues. Fig. 5 shows a result from one study circa 2010 [25]. Two solutions are shown for the CKM parameters $\rho^{\prime}$ and $\eta^{\prime}$. These are obtained by the overlap of two unitarity plane constraints. In each case the ratio of $\Delta M_{S}$ to $\Delta M_{d}$ is used. In one case the second constraint is that from $\varepsilon_{K}$ plus $\left|V_{c b}\right|$ and in the other case $S_{\psi K_{S}}$ is used. It's clear that a 100 effective event $K_{L} \rightarrow \pi^{0} v \bar{v}$ experiment could cleanly distinguish between the two cases.

Thus, we might find ourselves coming around full cycle to using the unique theoretical cleanliness of the ultra-rare kaon decays to determine the true value of the CKM parameters. And of course if there are small deviations from CKM, these decays can be used to pursue them to a level very hard to match in any other way. 


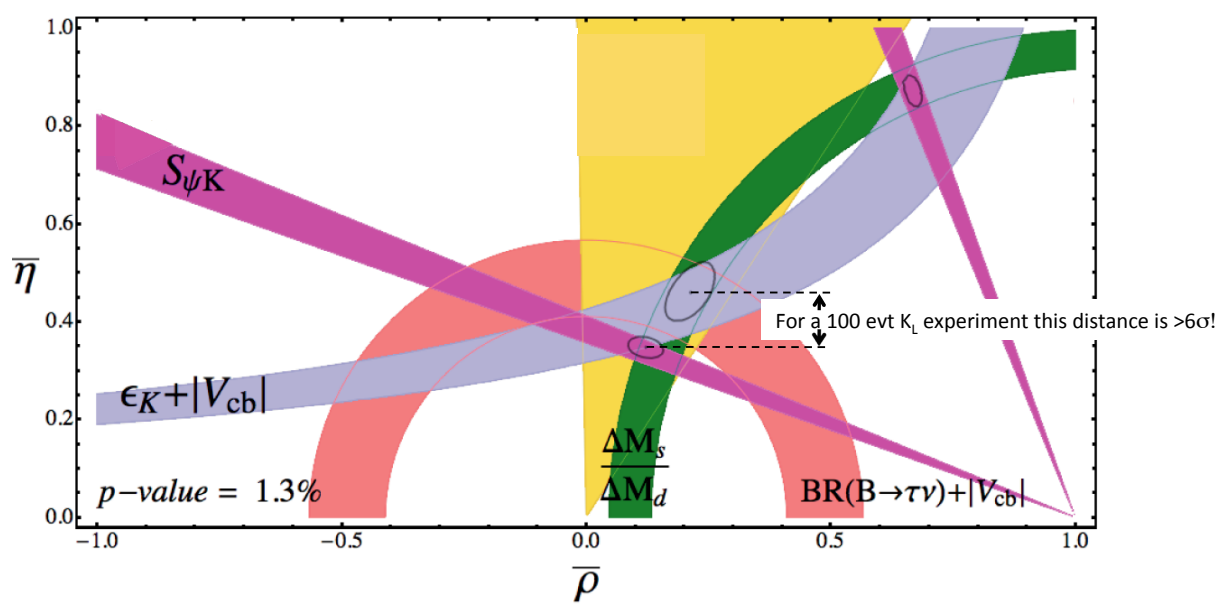

Figure 5: Adapted from Ref. [25], two solutions for $\rho^{\prime}$ and $\eta^{\prime}$, each uses $\frac{\Delta M_{s}}{\Delta M_{d}}$ as a constraint. For the second constraint, one uses $\varepsilon_{K}$ and the other $S_{\psi K_{S}}$. Note that distance between the $\eta^{\prime}$ of the two solutions corresponds to a $6 \sigma$ separation for a 100 effective event $K_{L} \rightarrow \pi^{0} v \bar{v}$ experiment.

\section{References}

[1] http://www-fars.nhtsa.dot.gov/Main/index.aspx

[2] D. M. Straub, arXiv:1012.3893 [hep-ph].

[3] S. Adler et al. [E949 and E787 Collaborations], Phys. Rev. D 77, 052003 (2008) [arXiv:0709.1000 [hep-ex]].

[4] J. K. Ahn et al. [E391a Collaboration], Phys. Rev. D 81, 072004 (2010) [arXiv:0911.4789 [hep-ex]].

[5] G. Anelli, A. Ceccucci, V. Falaleev, F. Formenti, A. Gonidec, B. Hallgren, P. Jarron and A. Kluge et al., CERN-SPSC-2005-013.

[6] T. Yamanaka [KOTO Collaboration], PTEP 2012, $02 \mathrm{~B} 006$ (2012).

[7] J. Comfort, D. Bryman, L. Doria, T. Numao, A. Sher, D. Vavilov, D. Jaffe and S. Kettell et al., FERMILAB-PROPOSAL-1021.

[8] D. Bryman, L. Littenberg http://project-X-kaons.fnal.gov/detector/K0_pnn_ProjX-1.pdf/view

[9] R. Appel, G. S. Atoian, B. Bassalleck, D. R. Bergman, D. N. Brown, N. Cheung, S. Dhawan and H. Do et al., Phys. Rev. Lett. 85, 2450 (2000) [hep-ex/0005016]. 
[10] http://www.bnl.gov/rsvp/KOPIO.htm

[11] J. W. Glenn, M. Brennan, L. Littenberg, J. Rose, C. Woody, A. Zaltsman, P. Bergbusch and D. Majka, Conf. Proc. C 970512, 967 (1997).

[12] G. S. Atoian, G. I. Britvich, S. K. Chernichenko, S. Dhawan, V. V. Issakov, O. V. Karavichev, T. L. Karavicheva and V. N. Marin et al., Nucl. Instrum. Meth. A 584, 291 (2008) [arXiv:0709.4514 [physics.ins-det]].

[13] J.R. Comfort et al., "KOPIO Project Conceptual Design Report”, April 2005, http://www.kopio.bnl.gov/cdr2005_nsf.pdf.

[14] A. J. Buras, G. Colangelo, G. Isidori, A. Romanino and L. Silvestrini, Nucl. Phys. B 566, 3 (2000) [hep-ph/9908371].

[15] J. R. Batley et al. [NA48/1 Collaboration], Phys. Lett. B 576, 43 (2003) [hep-ex/0309075].

[16] J. R. Batley et al. [NA48/1 Collaboration], Phys. Lett. B 599, 197 (2004) [hep-ex/0409011].

[17] F. Mescia, C. Smith and S. Trine, JHEP 0608, 088 (2006) [hep-ph/0606081].

[18] A. Alavi-Harati et al. [KTeV Collaboration], Phys. Rev. Lett. 93, 021805 (2004) [hep-ex/0309072].

[19] A. Alavi-Harati et al. [KTEV Collaboration], Phys. Rev. Lett. 84, 5279 (2000) [hep-ex/0001006].

[20] T. Alexopoulos et al. [KAMI Collaboration], FERMILAB-PROPOSAL-0922.

[21] D. Ambrose et al. [E871 Collaboration], Phys. Rev. Lett. 84, 1389 (2000).

[22] G. Isidori, C. Smith and R. Unterdorfer, Eur. Phys. J. C 36, 57 (2004) [hep-ph/0404127].

[23] L. Littenberg, hep-ex/0512044.

[24] R. Aaij et al. [LHCb Collaboration], JHEP 1301, 090 (2013) [arXiv:1209.4029 [hep-ex]].

[25] E. Lunghi and A. Soni, Phys. Lett. B 697, 323 (2011) [arXiv:1010.6069 [hep-ph]]. 